\title{
Thoracic epidural anesthesia in congenital heart surgery
}

\author{
Christopher Schmehil, MD, ${ }^{\mathrm{a}}$ Kyong-Jin Lee, MD, ${ }^{\mathrm{b}}$ Samuel Casella, MD, MPH, ${ }^{\mathrm{a}}$ and Daniel Millan, MD,
} Richmond, Va; Palo Alto, Calif; and San Antonio, Tex

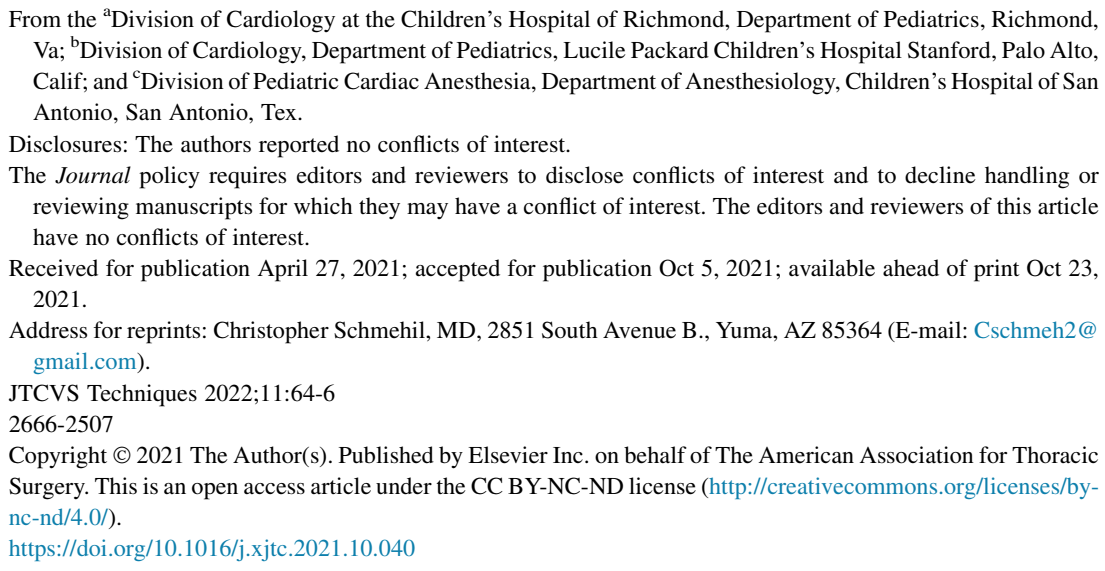

Thoracic epidural anesthesia (TEA) is widely applied in thoracic and abdominal surgeries, but its use in cardiac surgery remains controversial. ${ }^{1}$ Epidural anesthesia acts as a sympathetic blockade with varying effects on the cardiovascular system depending on the vertebral level of access. ${ }^{1,2}$ In addition to analgesia, other benefits of TEA may include early recovery of consciousness, shortened time to extubation, improved pulmonary function, hemodynamic stability, reduction of tachyarrhythmias, improved postoperative glucose control, and improved acute kidney function. ${ }^{1,3}$

Regardless of these benefits, the use of TEA in cardiothoracic surgery has been limited due to the potential complications of epidural hematoma formation and neurologic impairment. ${ }^{1}$ Despite the theoretically increased risk associated with anticoagulation, recent studies have estimated the risk of epidural hematoma formation in cardiac and thoracic surgical cases to be as low as 1:6628. ${ }^{4}$ Previous studies have shown an association with TEA insertion technique and duration of use with the development of epidural hematoma and/or abscess formation. ${ }^{5}$ Fluoroscopic- and ultrasound-guided epidural catheter placement are methods by which procedural success and safety may be increased by direct visualization of the bony structures, spinal level, and epidural space during insertion (Figure 1). Since 2015, fluoroscopy-guided TEA (fTEA) has become the standard of care for our cardiac surgical patients, including newborns (Figure 2). We describe the technique and outcomes associated with fTEA at our institution.

\section{METHODS}

We performed a retrospective chart review for all patients from 0 to 30 years of age who underwent congenital heart surgery with attempted fTEA at our institution from 2015 to 2020 . The primary outcome was successful placement of an epidural catheter. Secondary outcomes included
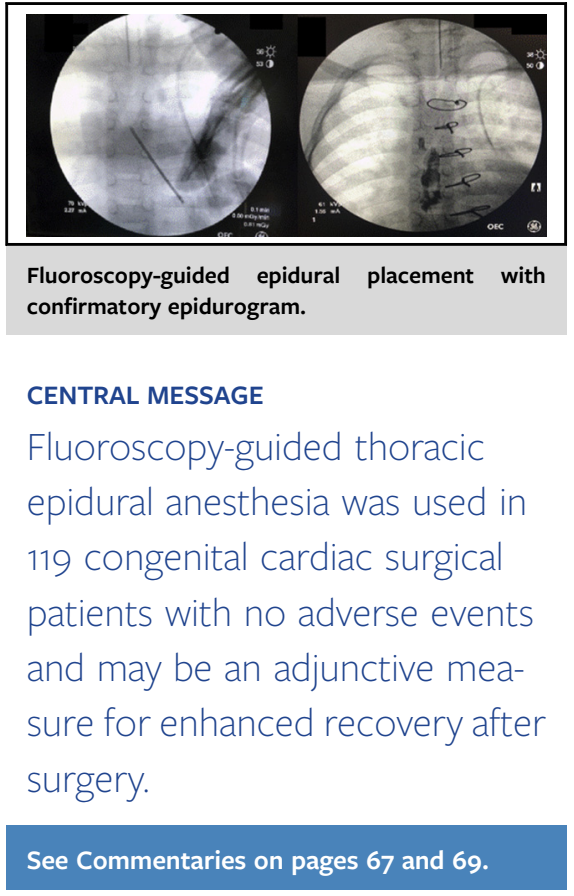

complications (epidural hematoma, abscess, and/or neurologic complication), time to extubation, and length of stay.

All epidurals were performed by the pediatric cardiac anesthesiologist under fluoroscopic guidance. Epidural anesthetics typically comprised clonidine and ropivacaine.

\section{RESULTS}

In total, 120 patients underwent congenital cardiac surgery with attempted fTEA at our institution. The median age and weight at time of surgery was 24 months (range, 3 days-27 years) and $11.0 \mathrm{~kg}$ (range, 2.1-113.0), respectively. Cardiac diagnoses are listed in Table 1. fTEA was successful in $119(99 \%)$ patients. fTEA was aborted in 1 patient due to poor visualization of the spine related to scoliosis and the presence of Harrington rods. There were no observed complications during the study time period. Of the 119 patients who had fTEA placed, 110 patients $(92 \%)$ were extubated in the operating room. The median duration of epidural and length of stay were 2 days (range, 1-29) and 3 days (range, 2-48), respectively.

\section{Example Patient}

A 3.3-kg neonate with D-looped transposition of the great arteries underwent an arterial switch operation at 8 days of life. Uncomplicated fTEA was placed before the start of the case with the epidural catheter at the T3-T4 thoracic spinal 


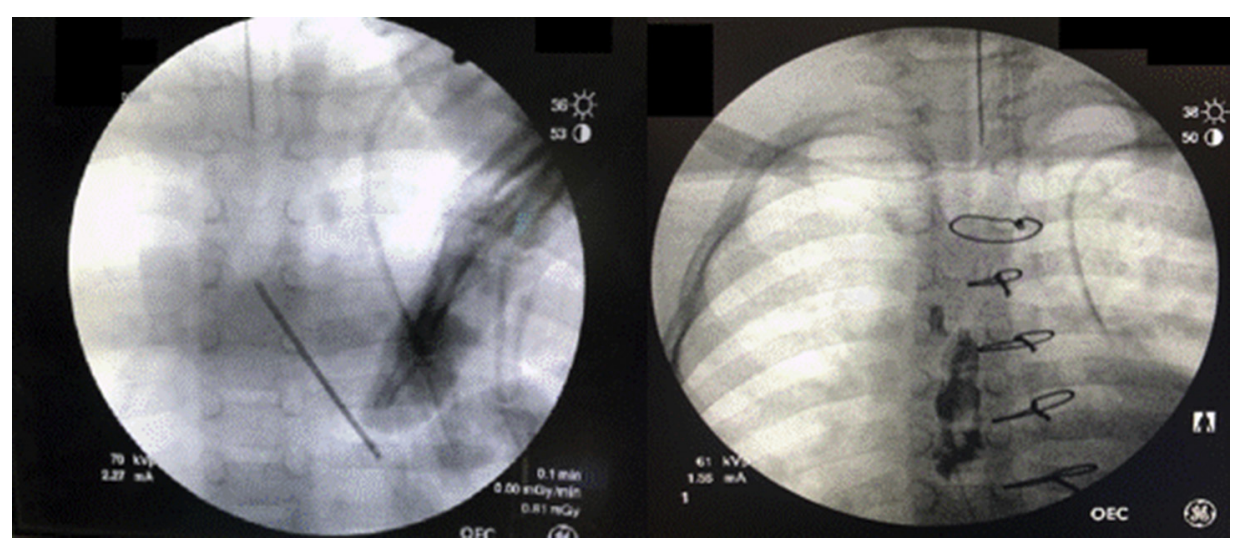

FIGURE 1. Fluoroscopy-guided epidural placement with confirmatory epidurogram.

level. The surgery, performed on cardiopulmonary bypass, was uncomplicated, and the patient was successfully extubated in the operating room. The epidural catheter was removed on postoperative day 3 and the patient was discharged on postoperative day 5 .

The institutional review board (IRB) waived the need for ethics approval and the need to obtain consent for the collection, analysis, and publication of the retrospectively obtained and anonymized data for this non-interventional study (IRB approval number: HM20019284 Ame1; IRB approval date: December 14, 2020).

\section{DISCUSSION}

The current study found no adverse events in 119 patients who underwent successful epidural anesthesia during congenital heart surgery. With a recent focus and commitment toward Enhanced Recovery After Surgery programs, our experience would support investigating the use of fTEA as an adjunctive measure for expedited recovery.

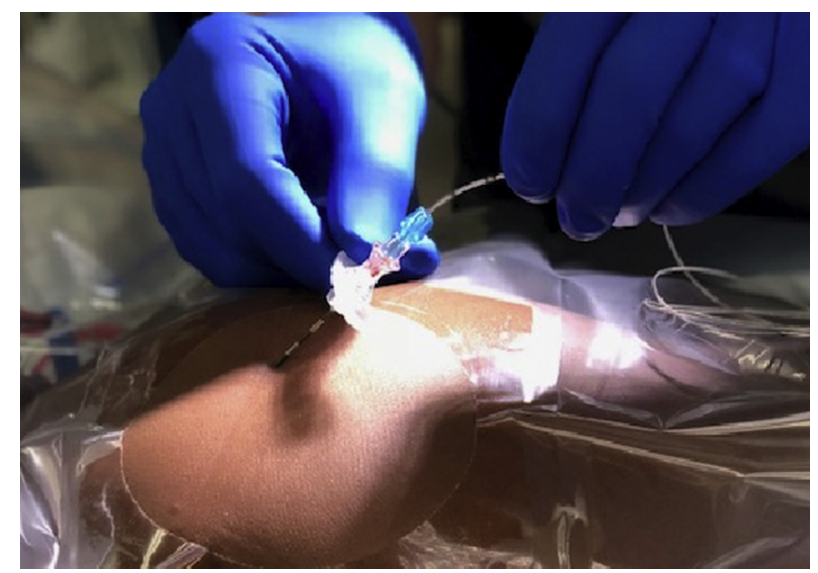

FIGURE 2. After the patient is placed in the prone position, the epidural catheter is advanced through the Tuohy needle and the tip of the catheter can be seen fluoroscopically. Epidurogram is performed to confirm location. The epidural catheter is ideally placed at the T3-T4 spinal level.
Enhanced Recovery After Surgery protocols have resulted in shorter lengths of stay by $30 \%$ to $50 \%$ with similar reductions in complications, readmissions, and costs. ${ }^{6}$ There appear to be potential benefits of TEA, but its use has been limited in our population due to the risk of epidural hematoma, abscess formation, and neurologic complications. We acknowledge the small size of the present study, and although there were no observed adverse events, we would advise adhering to the precautions recommended by the American Society of Regional Anesthesia in order to minimize the risk. ${ }^{7}$ fTEA may be a method by which these complications can be avoided enabling its use in day-to-day practice. This retrospective review is the first to our knowledge that supports the use of fTEA in congenital cardiac surgical patients. This may in turn allow for future studies comparing fTEA to other methods of sedation and anesthesia, including non-neuraxial anesthesia, in an effort to develop safe and effective modifications to our standards of patient care.

TABLE 1. Patient characteristics $(\mathbf{n}=\mathbf{1 2 0})$

\begin{tabular}{lc}
\hline \multicolumn{2}{c}{ Demographics } \\
\hline Age, mo & 24 (0.1-324) \\
Weight, kg & 11 (2.1-113) \\
Male:female & $72: 48$ \\
Diagnosis, $n$ & \\
Arch anomaly & 6 \\
Atrial and/or ventricular septal defect & 33 \\
Atrioventricular canal defect & 22 \\
Atrioventricular valve anomaly & 4 \\
Conotruncal anomaly & 29 \\
LVOT anomaly & 5 \\
Patent ductus arteriosus & 2 \\
Single ventricle & 9 \\
Transposition of the great arteries & 4 \\
Other & 6 \\
\hline
\end{tabular}

Values are expressed as median (range) for continuous variables. LVOT, Left ventricular outflow tract. 


\section{References}

1. Jakobsen CJ. High thoracic epidural in cardiac anesthesia: a review. Semin Cardiothorac Vasc Anesth. 2015;19:38-48.

2. Wink J, Veering BT, Aarts L, Wouters PF. Effects of thoracic epidural anesthesia on neuronal cardiac regulation and cardiac function. Anesthesiology. 2019;130:472-91.

3. Chakravarthy M, Thimmangowda P, Krishnamurthy J, Nadiminti S, Jawali V. Thoracic epidural anesthesia in cardiac surgical patients: a prospective audit of 2,113 cases. J Cardiothorac Vasc Anesth. 2005;19:44-8.

4. Hemmerling TM, Cyr S, Terrasini N. Epidural catheterization in cardiac surgery. The 2012 risk assessment. Ann Card Anaesth. 2013;16:169-77.
5. Wang LP, Hauerberg J, Schmidt JF. Incidence of spinal epidural abscess after epidural analgesia: a national 1-year survey. Anesthesiology. 1999; 91:1928-36

6. Ljungqvist $\mathrm{O}$, Scott M, Fearon KC. Enhanced recovery after surgery: a review. JAMA Surg. 2017;152:292-8.

7. Horlocker T, Vandermeuelen E, Kopp S, Gogarten W, Leffert LR, Benzon HT. Regional anesthesia in the patient receiving antithrombotic or thrombolytic therapy. American Society of Regional Anesthesia and Pain Medicine evidence-based guidelines (fourth edition). Reg Anesth Pain Med. 2018;43: 263-309. 